\title{
Anomalies of geomagnetic field due to a vertical prolate rotational ellipsoid
}

\author{
Milan HVOŽDARA ${ }^{1}$, Ján VOZÁR ${ }^{1,2}$ \\ ${ }^{1}$ Geophysical Institute of the Slovak Academy of Sciences \\ Dúbravská cesta 9, 84528 Bratislava, Slovak Republic; e-mail: geofhvoz@savba.sk \\ ${ }^{2}$ Dublin Institute for Advanced Studies; e-mail: vozar@cp.dias.ie
}

\begin{abstract}
We present an exact analytical solution of the forward magnetometric problem for the perturbing body having the shape of the rotational prolate ellipsoid with the longer axis oriented vertically to the surface of the earth. The anomaly of $\Delta Z$ and $\Delta T$ is calculated for the network of points in the plane $z=$ const above the ellipsoid, as well as for the points on the surfaces of the volcanic hill: i) the cut cone, ii) the smooth shape given by the rotation of the Gaussian curve. Theoretical results can be useful for the interpretation of land or aeromagnetic survey in the volcanic areas.
\end{abstract}

Key words: geomagnetic anomalies models, potentials of prolate spheroid, magnetometry in volcanic areas

\section{Introduction}

The forward magnetometric problem for the rotational prolate ellipsoid is in the interest of applied magnetometry in some volcanic and post volcanic regions. This body can be used as a model of magmatic intrusions below the main crater. For the solution of this problem we will use classical method of separation of variables in the curvilinear prolate ellipsoidal coordinate system. Similar potential problems were solved for static electric field (Smythe, 1968) and also in geoelectric potential field (Cook and Nostrand, 1982; Wait, 1982). We used this treatment in the solution of groundwater flow anomalies due to a oblate spheroidal body (Hvoždara, 2009). We will calculate the magnetic induction anomaly considering magnetic permeability of the body to be uniform:

$\mu_{T}=\mu_{0}(1+\kappa)$, 
where $\mu_{0}$ is the magnetic permeability of the vacuum and $\kappa$ is the magnetic susceptibility of the intrusion. It is clear that for the hot magma with temperature over the Curie point we put $\kappa \rightarrow 0$, but for cooled solidified magmatic body we put $\kappa$ valid for basalts or andesites $\kappa \approx 0.01-0.1$ (in SI system).

\section{Formulation of the problem}

Let us consider the perturbing body in the form of uniform prolate spheroid bounded by rotation of the ellipse with semiaxes $a, b(a>b)$ around the vertical axis of symmetry $z$, which prolongate the vertical semiaxis $a$ downward as shown in Fig. 1 (section in the $x, z$ plane). The section of this spheroid by the horizontal plane $(x, y)$ is the circle $x^{2}+y^{2} \leq b^{2}$. Let the unperturbed magnetic field far from the spheroid be uniform with induction

$\boldsymbol{B} \equiv\left(X_{0}, Y_{0}, Z_{0}\right)$,

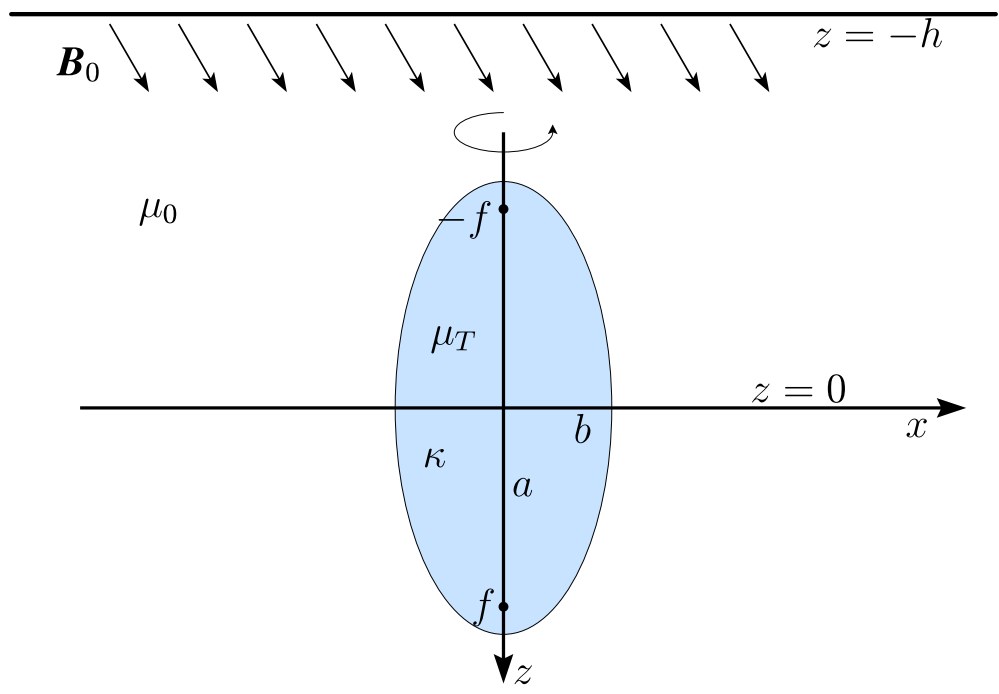

Fig. 1. Model of a prolate rotationally symmetric spheroid. 
where $X_{0}, Y_{0}, Z_{0}$ are its components in the local Cartesian coordinate system. We can write its potential in the form:

$U_{0}=-B_{0}\left(x \cos \varphi_{0} \cos I+y \sin \varphi_{0} \cos I+z \sin I\right)$,

where $I$ is the geomagnetic inclination angle and $\varphi_{0}$ is the declination angle with respect to the geographic North. Here $B_{0}$ is the modulus (total induction) of the magnetic field $\boldsymbol{B}_{0}$ :

$B_{0}=\left(X_{0}^{2}+Y_{0}^{2}+Z_{0}^{2}\right)^{1 / 2}, \mathrm{nT}$.

The magnetic field in our problem is time steady, so it satisfies Maxwell equations

$\operatorname{rot} \boldsymbol{B}=0, \quad \operatorname{div} \boldsymbol{B}=0$,

hence we can represent it by using the magnetic potential $U(x, y, z)$ :

$\boldsymbol{B}=-\operatorname{grad} U$.

It is clear that potential obeys the Laplace equation:

$\operatorname{div} \operatorname{grad} U=0$.

We denote the potential inside the spheroid by $U_{T}$ and outside it as $U_{1}=$ $U_{0}+U_{1}^{*}$, where $U_{1}^{*}$ is the perturbing potential outside the body. On the surface $S$ of the spheroid the following boundary conditions must be satisfied:

$\mu_{r}\left[U_{0}+U_{1}^{*}\right]_{S}=\left[U_{T}\right]_{S}$,

$\left[\frac{\partial}{\partial n}\left(U_{0}+U_{1}^{*}\right)\right]_{S}=\left[\frac{\partial}{\partial n} U_{T}\right]_{S}$.

These follow from the continuity of the tangential component of magnetic intensity $\boldsymbol{H}=\mu^{-1} \boldsymbol{B}$ and of the normal component of $\boldsymbol{B}$. Here we introduce relative permeability of the perturbing body:

$\mu_{r}=\mu_{T} / \mu_{0}=1+\kappa$.

As we anticipated, we will solve this boundary value problem by the separation of variables in the prolate spheroidal system $(\alpha, \beta, \varphi)$. The transformation relations to the Cartesian system are: 
$x=f \operatorname{sh} \alpha \sin \beta \cos \varphi, \quad y=f \operatorname{sh} \alpha \sin \beta \sin \varphi, \quad z=f \operatorname{ch} \alpha \cos \beta$,

(see e.g. Lebedev, 1963; Arfken, 1966; Madelung, 1957). The curvilinear coordinates $\alpha, \beta, \varphi$ vary over the intervals:

$\alpha \in\langle 0,+\infty), \quad \beta \in\langle 0, \pi\rangle, \quad \varphi=\langle 0,2 \pi\rangle$,

and $f$ is the prolatness parameter

$f=\sqrt{a^{2}-b^{2}}$,

where $a, b$ are the lengths of major or minor semiaxes, respectively, for the generating vertical ellipse. Using transformation relations (10) we can find that the surfaces $\alpha=$ const are $z$-prolongated ellipsoids defined by equations:

$\frac{x^{2}+y^{2}}{f^{2} \operatorname{sh}^{2} \alpha}+\frac{z^{2}}{f^{2} \operatorname{ch}^{2} \alpha}=1 \quad$ or $\quad \frac{r^{2}}{f^{2} \operatorname{sh}^{2} \alpha}+\frac{z^{2}}{f^{2} \operatorname{ch}^{2} \alpha}=1$,

where $r=\sqrt{x^{2}+y^{2}}$ is the horizontal distance from $z$-axis. The contour of the ellipse in the plane $(x, z)$ which generates the surface of the $z$-prolate spheroid $S$ is:

$x^{2} / b^{2}+z^{2} / a^{2}=1$.

This surface is matched with the surface of the supporting spheroid $\alpha=\alpha_{0}$ if we put

$f^{2} \operatorname{sh}^{2} \alpha_{0}=b^{2}, \quad f^{2} \operatorname{ch}^{2} \alpha_{0}=a^{2}$.

Then we obtain the prolatness parameter $f$ :

$f^{2}=a^{2}-b^{2}, \quad f=\sqrt{a^{2}-b^{2}}$,

which means that $f$ is the numerical eccentricity of the ellipse with foci on the $z$ axis: $(0,0,-f),(0,0,+f)$. The polar axis for the angle $\beta$ is the $z$ axis (pointing downward), while the halfline $z \in(0,+\infty)$ which corresponds to $\beta=0$ and the halfline $z \in(0,-\infty)$ which corresponds to $\beta=\pi$. The coordinate surfaces $\beta=$ const are hyperboloids:

$\frac{z^{2}}{f^{2} \cos ^{2} \beta}-\frac{r^{2}}{f^{2} \sin ^{2} \beta}=1$ 
these are orthogonal and confocal to ellipsoids $\alpha=$ const. Using equations (14) we obtain important relations for calculation of the parameter $\alpha$ :

$e^{\alpha_{0}}=(a+b) / f, \quad \alpha_{0}=\ln [(a+b) / f]$.

In this manner we can link the geometrical parameters of the spheroid to the curvilinear coordinates. It is clear, that normal derivatives in formula (8) correspond to $\partial / \partial \beta$ since surfaces $\beta=$ const are orthogonal to surfaces $\alpha=$ const. We will also use Lame's metric parameters given e.g. by (Lebedev, 1963):

$h_{\alpha}=f\left(\operatorname{sh}^{2} \alpha+\sin ^{2} \beta\right)^{1 / 2}, \quad h_{\varphi}=f \operatorname{sh} \alpha \sin \beta$,

which we will use in calculations of $\operatorname{grad} U$. The particular solution of Laplace equation in prolate ellipsoidal system can be found e.g. in Lebedev, 1963; Morse and Feshbach, 1953. This is a combination of spherical functions:

$U_{n m}(\alpha, \beta, \varphi)=\left\{\begin{array}{l}P_{n}^{m}(\operatorname{ch} \alpha) \\ Q_{n}^{m}(\operatorname{ch} \alpha)\end{array}\right\} P_{n}^{m}(\cos \beta)\left\{\begin{array}{c}\cos m \varphi \\ \sin m \varphi\end{array}\right\}$.

Here $P_{n}^{m}(s), Q_{n}^{m}(s)$ are the associated spherical functions for real argument $s=\operatorname{ch} \alpha>1, P_{n}^{m}(\cos \beta)$ are associated Legendre functions of degree $n$, order $m$ :

$P_{n}^{m}(\eta)=\left(1-\eta^{2}\right)^{m / 2} \frac{\mathrm{d}^{m} P_{n}(\eta)}{\mathrm{d} \eta^{m}}$,

where $\eta=\cos \beta \in<-1,+1>$. From the theory we know that spherical functions of the second kind $Q_{n}^{m}(s)$ are singular for $s \rightarrow 1$, so in the interior potential $U_{T}(\alpha, \beta, \varphi)$ cannot be used. The unperturbed potential field $U_{0}$ given by (3) can be written in the form:

$$
\begin{aligned}
U_{0}(\alpha, \beta, \varphi) & =-B_{0} r \cos I \cos \left(\varphi-\varphi_{0}\right)-z \sin I B_{0}= \\
& =-B_{0} f \operatorname{sh} \alpha \sin \beta \sin I \cos \left(\varphi-\varphi_{0}\right)-B_{0} f \sin I \operatorname{ch} \alpha \cos \beta= \\
& =-D_{0} f \operatorname{ch} \alpha \cos \beta-E_{0} f \operatorname{sh} \sin \beta \cos \left(\varphi-\varphi_{0}\right),
\end{aligned}
$$

where

$E_{0}=B_{0} \cos I, \quad D_{0}=B_{0} \sin I$, 
are horizontal and vertical components of $\boldsymbol{B}_{0}$. This primary potential contains azimuthal dependence $\cos \left(\varphi-\varphi_{0}\right)$ in the second term and is $\varphi$ independent in the first term. This will be true also for $U_{T}$ and $U_{1}^{*}$. Similar property will occur also for $\beta$ dependence, since $\sin \beta=P_{1}^{1}(\cos \beta)$ and $\cos \beta=P_{1}(\cos \beta)$. Then we construct the interior potential $U_{T}(\alpha, \beta, \varphi)$ in the form as a multiplier of the primary potential terms:

$U_{T}(\alpha, \beta, \varphi)=-D_{0} a_{2} f \operatorname{ch} \cos \beta-E_{0} g_{2} f \operatorname{sh} \alpha \sin \beta \cos \left(\varphi-\varphi_{0}\right)$.

The potential outside the spheroid we consider as the sum of $U_{0}$ and perturbing part $U_{1}^{*}$ which must decrease for $\cosh \alpha \rightarrow+\infty$, so it cannot contain $P_{n}^{m}(\cosh \alpha)$. Then we have:

$$
\begin{aligned}
U_{1}(\alpha, \beta, \varphi)= & -D_{0} f\left[\operatorname{ch} \alpha+a_{1} Q_{1}(\operatorname{ch} \alpha)\right] \cos \beta- \\
& -E_{0} f\left[\operatorname{sh} \alpha+g_{1} Q_{1}^{1}(\operatorname{ch} \alpha)\right] \sin \beta \cos \left(\varphi-\varphi_{0}\right) .
\end{aligned}
$$

Coefficients $a_{1}, g_{1}, a_{2}, g_{2}$ we determine from boundary conditions (7), (8) where the normal derivative we take as $\partial / \partial \alpha$. We use orthogonality of goniometric functions $\sin \beta, \cos \beta$ as well as $\cos \varphi, \sin \varphi$. Then we obtain two pairs of equations for $m=0$ and $m=1$. After easy modifications we obtain for $\alpha=\alpha_{0}$ :

$\mu_{r}\left[\operatorname{ch} \alpha_{0}+a_{1} Q_{1}\left(\operatorname{ch} \alpha_{0}\right)\right]=a_{2} \operatorname{ch} \alpha_{0}, \quad 1+a_{1} Q_{1}^{\prime}\left(\operatorname{ch} \alpha_{0}\right)=a_{2}$,

$\mu_{r}\left[\operatorname{sh} \alpha_{0}+g_{1} Q_{1}^{1}\left(\operatorname{ch} \alpha_{0}\right)\right]=g_{2} \operatorname{sh} \alpha_{0}, \quad \operatorname{ch} \alpha_{0}+g_{1} \operatorname{sh} \alpha_{0} Q_{1}^{1^{\prime}}\left(\operatorname{ch} \alpha_{0}\right)=g_{2} \operatorname{ch} \alpha_{0} \cdot(26)$

We can easily solve these equations by elimination method and obtain the following expressions for the pair $\left(a_{1}, a_{2}\right)$, using the abbreviation $t_{0}=\operatorname{ch} \alpha_{0}$ :

$$
\begin{aligned}
& a_{1}=\frac{\left(\mu_{r}-1\right) t_{0}}{t_{0} Q_{1}^{\prime}\left(t_{0}\right)-\mu_{r} Q_{1}\left(t_{0}\right)}, \\
& a_{2}=\frac{\mu_{r}\left[t_{0} Q_{1}^{\prime}\left(t_{0}\right)-Q_{1}\left(t_{0}\right)\right]}{t_{0} Q_{1}^{\prime}\left(t_{0}\right)-\mu_{r} Q_{1}\left(t_{0}\right)} .
\end{aligned}
$$

The second pair $\left(g_{1}, g_{2}\right)$ will be:

$g_{1}=\frac{t_{0}\left(\mu_{r}-1\right) \sqrt{t_{0}^{2}-1}}{\left(t_{0}^{2}-1\right) Q_{1}^{1^{\prime}}\left(t_{0}\right)-\mu_{r} t_{0} Q_{1}^{1}\left(t_{0}\right)}$, 
$g_{2}=\mu_{r} \frac{\left(t_{0}^{2}-1\right) Q_{1}^{1^{\prime}}\left(t_{0}\right)-t_{0} Q_{1}^{1}\left(t_{0}\right)}{\left(t_{0}^{2}-1\right) Q_{1}^{1^{\prime}}\left(t_{0}\right)-\mu_{r} t_{0} Q_{1}^{1}\left(t_{0}\right)}$

where we used $t_{0}^{2}-1=\operatorname{sh}^{2} \alpha_{0}$. Verification of these coefficients for the case of non-magnetic spheroid $\mu_{r} \rightarrow 1$ will give zero value for the perturbing potential $U_{1}^{*}$ given by multiplier coefficients $a_{1}$ and $g_{1}$ and in this case the interior potential $U_{T}$ will be equal to the exciting one $U_{0}$. Since the spherical functions of the 2nd kind $Q_{1}(t), Q_{1}^{1}(t)$ are not very common in geophysics we quote expressions for these functions valid for $t>1$. According to theory given by Lebedev (1963) or Arfken (1966), we have the representations:

$$
\begin{aligned}
& Q_{1}(t)=\sum_{k=0}^{\infty} \frac{1}{2 k+3} \frac{1}{t^{2 k+2}}=(t / 2) \ln \frac{t+1}{t-1}-1, \\
& Q_{1}^{1}(t)=\left(t^{2}-1\right)^{1 / 2} \frac{\mathrm{d}}{\mathrm{d} t} Q_{1}(t)= \\
& =-\left(t^{2}-1\right)^{1 / 2} \sum_{k=0}^{\infty} \frac{2 k+2}{2 k+3} \frac{1}{t^{2 k+3}}=\left(t^{2}-1\right)^{1 / 2}\left[\frac{1}{2} \ln \frac{t+1}{t-1}-\frac{t}{t^{2}-1}\right] .
\end{aligned}
$$

The expressions for their derivatives $Q_{1}^{\prime}(t), Q_{1}^{1^{\prime}}(t)$ can be easily obtained by the derivation of series in (31), (32). It can be easily verified that all these functions decrease to zero for $t \rightarrow+\infty$.

\section{Calculation of the anomalous magnetic field}

We have obtained coefficients which modify the unperturbed magnetic field potential inside and outside the prolate spheroidal perturbing body. The magnetic field potential inside the body (24) can be easily expressed in the Cartesian coordinates $(x, y, z)$ using transformation relations (10) and (22):

$U_{T}(x, y, z)=-x B_{0} a_{2} \sin I \cos \varphi_{0}-y B_{0} a_{2} \cos I \sin \varphi_{0}-z B_{0} g_{2} \sin I$.

This represent potential of the directional uniform magnetic field:

$\boldsymbol{B}_{T} \equiv\left(B_{0} a_{2} \cos I \cos \varphi_{0}, \quad B_{0} a_{2} \cos I \sin \varphi_{0}, \quad B_{0} g_{2} \sin I\right)$. 
We can see that the horizontal declination angle $\varphi_{0}$ will be preserved in the spheroid (clearly due to axial symmetry), but the inclination angle will be changed to $I_{T}$, which can be calculated from the relation:

$\operatorname{tg} I_{T}=\left(g_{2} / a_{2}\right) \operatorname{tg} I$.

From the practical point of view the most interesting is the anomalous magnetic field outside the spheroid. Its potential can be obtained from (24) in the form

$U_{1}^{*}(\alpha, \beta, \varphi)=-D_{0} f a_{1} Q_{1}(\operatorname{ch} \alpha) \cos \beta-E_{0} f g_{1} Q_{1}^{1}(\operatorname{ch} \alpha) \sin \beta \cos \left(\varphi-\varphi_{0}\right)$.

We can see that the first part, proportional to $D_{0} a_{1}$, is independent of $\varphi$ (it is axially symmetric), while the second one is azimutally dependent via $\cos \left(\varphi-\varphi_{0}\right)$, where $\varphi_{0}$ is the declination angle of the $B_{0}$. The perturbing magnetic field outside the spheroid can be calculated from the curvilinear components of the $-\operatorname{grad} U_{1}^{*}(\alpha, \beta, \varphi)$, i.e.:

$B_{\alpha}^{*}=-\frac{1}{h_{\alpha}} \frac{\partial U_{1}^{*}}{\partial \alpha}, \quad B_{\beta}^{*}=-\frac{1}{h_{\beta}} \frac{\partial U_{1}^{*}}{\partial \beta}, \quad B_{\varphi}^{*}=-\frac{1}{h_{\varphi}} \frac{\partial U_{1}^{*}}{\partial \varphi}$,

where the Lame's metrical parameters are given by (18). These curvilinear components can be easily transformed to the Cartesian components using formulae:

$$
\begin{aligned}
& B_{x}^{*}=B_{r}^{*} \cos \varphi-B_{\varphi}^{*} \sin \varphi, \quad B_{y}^{*}=B_{r}^{*} \sin \varphi+B_{\varphi}^{*} \cos \varphi, \\
& B_{z}^{*}=\left[-B_{\beta}^{*} \operatorname{ch} \alpha \sin \beta+B_{\alpha}^{*} \operatorname{sh} \alpha \cos \beta\right]\left[\operatorname{sh}^{2} \alpha+\sin ^{2} \beta\right]^{-1 / 2}, \\
& B_{r}^{*}=\left[-B_{\beta}^{*} \operatorname{sh} \alpha \cos \beta+B_{\alpha}^{*} \operatorname{ch} \alpha \sin \beta\right]\left[\operatorname{sh}^{2} \alpha+\sin ^{2} \beta\right]^{-1 / 2} .
\end{aligned}
$$

Here we adopted the formulae from Madelung (1957) with substitution $(v, u, \varphi) \rightarrow(\alpha, \beta, \varphi), u=\pi / 2-\beta$ and Madelung's $a_{u}=-B_{\beta}$ because the direction of his unit vector $\boldsymbol{e}_{u}$ is opposite to our $\boldsymbol{e}_{\beta}$. Because the calculation of the anomalous magnetic field outside the spheroid needs to be performed on some network of $(x, y, z)$ coordinates, we must use transformation relations to the curvilinear $(\alpha, \beta, \varphi)$ for the prolate spheroid. The treatment presented in Hvoždara (2009) gives relations

$2 f \operatorname{ch} \alpha=\left[r^{2}+(z-f)^{2}\right]^{1 / 2}+\left[r^{2}+(z+f)^{2}\right]^{1 / 2}$, 
where $r^{2}=x^{2}+y^{2}$. We have also $\operatorname{sh} \alpha=\left[\operatorname{ch}^{2} \alpha-1\right]^{1 / 2}$ and hence

$e^{\alpha}=\operatorname{ch} \alpha+\operatorname{sh} \alpha$.

For the angle coordinate $\beta$ we have:

$\cos \beta=z /(f \operatorname{ch} \alpha)$,

for the upper halfspace $z<0$ we have clearly $\beta \in(\pi / 2, \pi\rangle$. The azimutal angle $\varphi$ is given by the relation

$\operatorname{tg} \varphi=y / x, \quad \varphi \in\langle 0,0,2 \pi\rangle$.

Now we have a complete set of theoretical formulae for numerical calculations.

\section{Numerical calculations and discussion}

For the numerical calculations we choose prolate spheroid with semiaxes $a=500 \mathrm{~m}, b=200 \mathrm{~m}$ and susceptibility $\kappa=0.1$. For practical needs there are interesting model calculations of the $\Delta Z$ and $\Delta T$ anomaly for the case of aeromagnetic survey in a given plane $z=-H_{0}$ above the magmatic body or for land profile measurements on the hill above the magmatic body. In this case we have $z=-H(x, y)$. We will consider also two types of the axially symmetric hills, as defined later.

The results for the case of constant level $z_{p}=-2 a=-1000 \mathrm{~m}$ are given in Figs. 2a-c. In Fig. 2a we have plotted isolines of perturbing potential $U^{*}\left(x, y, z_{p}\right)$ divided by $B_{0}$. These can be characterized like the potential due to some inclined magnetic dipole, with the negative $(-)$ pole in the halfplane $x / a<0$ and close to the surface. The positive $(+)$ pole is deeper than negative one and lies in the halfplane $x / a>0$. For better clarity the profile curve of $U^{*}$ along the $x$ profile for $y_{c}=0$ is also presented. Figure $2 \mathrm{~b}$ presents isolines of $\Delta Z$ normed by $B_{0}$ and Fig. $2 \mathrm{c}$ isolines of $\Delta T\left(x, y, z_{p}\right)$. The relative values of $\Delta Z / B_{0}$ and $\Delta T / B_{0}$ are multiplied by the factor 1000. We can see that if $B_{0}=50000 \mathrm{nT}$, then maximum of $\Delta T$ will be around $60 \mathrm{nT}$, while minimum is about $-15 \mathrm{nT}$. We note that we consider the declination angle $D_{0} \equiv \varphi_{0}=0^{\circ}$ and inclination $I_{0}=55^{\circ}$, which 


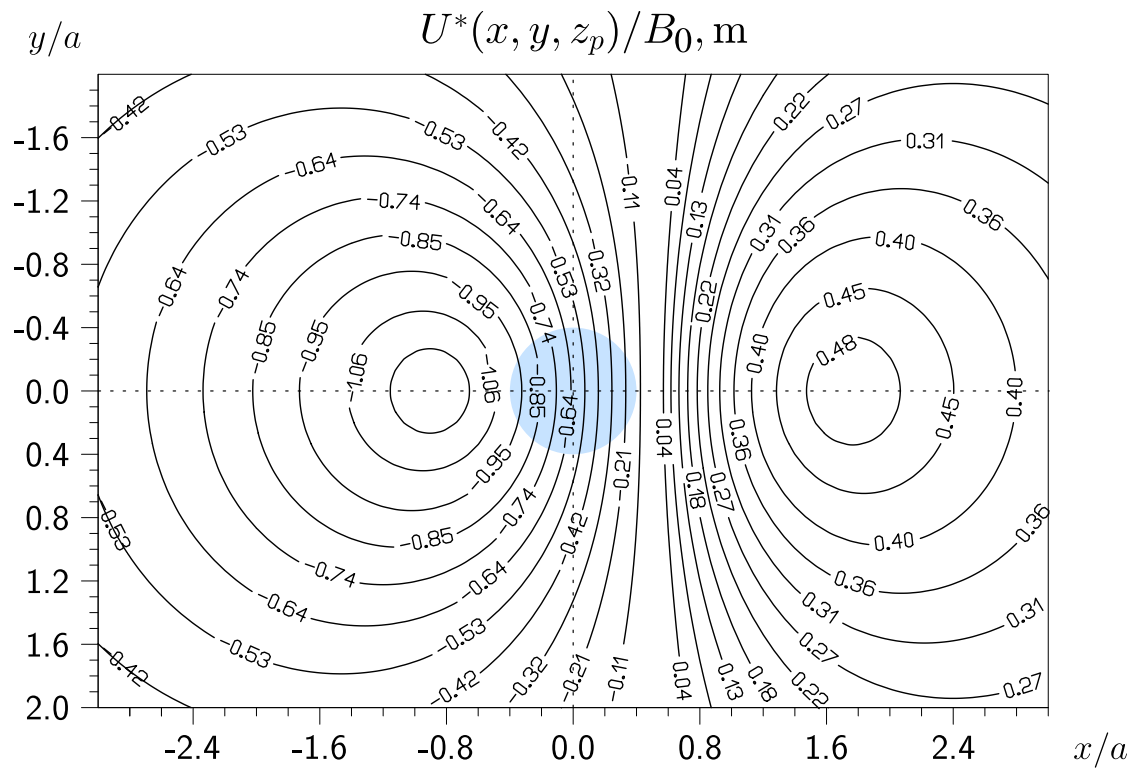

$U^{*}\left(x, 0, z_{p}\right) / B_{0}, \mathrm{~m}$

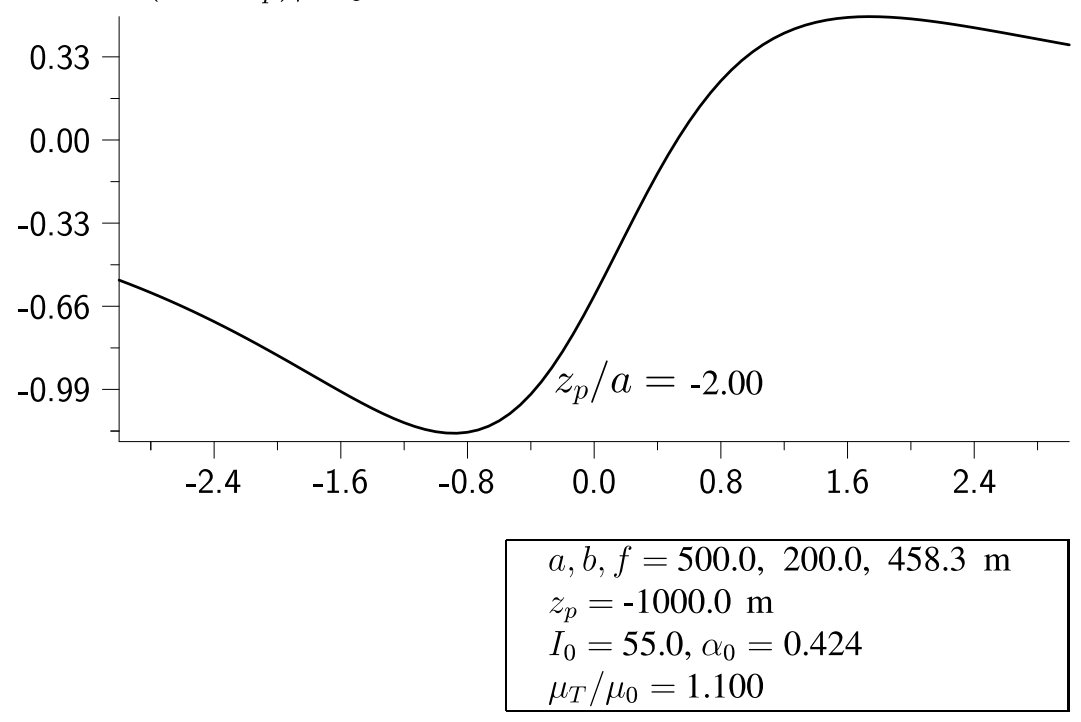

Fig. 2a. Isolines of the anomalous potential above the prolate spheroidal body at the plane $z_{p}=-2 a$. The bottom curve presents values along the profile $y=0$. 

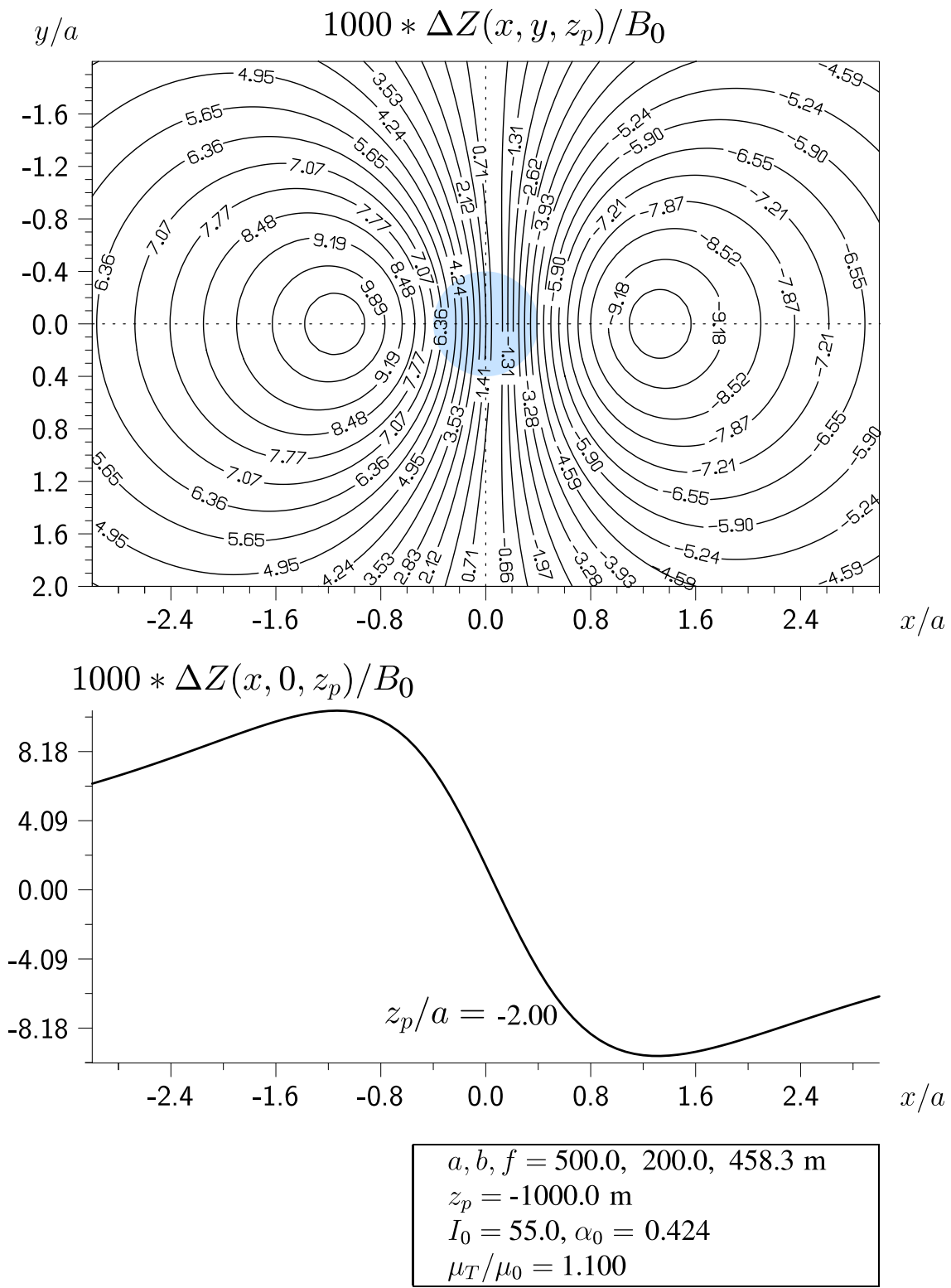

Fig. 2b. The isolines of the relative $\Delta Z$ anomaly above the prolate spheroidal body at the level $z_{p}=-2 a$. The bottom curve shows the profile values at $y=0$ for the level $z_{p} / a=-2$. 

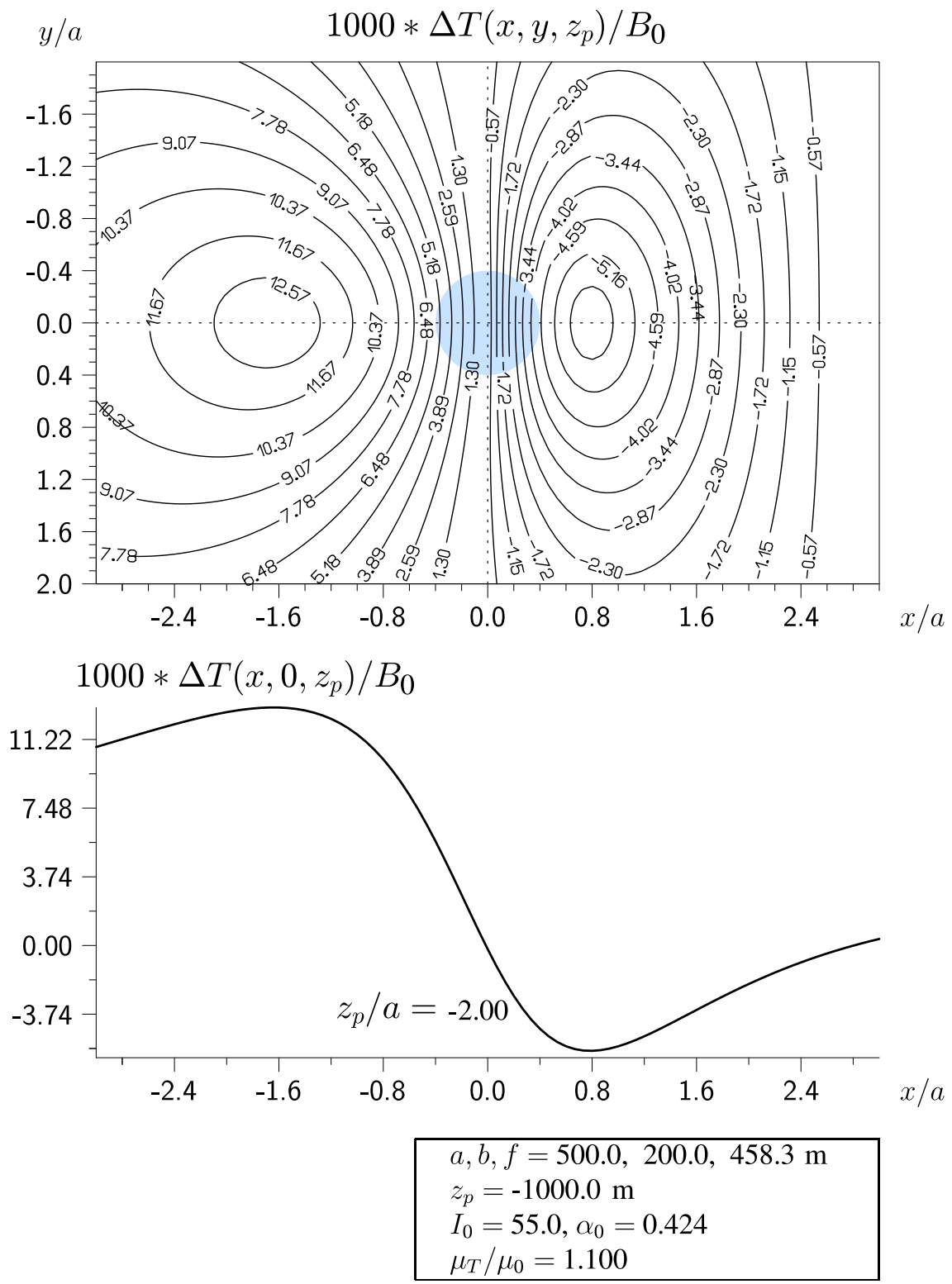

Fig. 2c. The isolines of the relative $\Delta T$ anomaly above the prolate spheroidal body at the level $z_{p}=-2 a$. The bottom curve shows the profile values at $y=0$ for the level $z_{p} / a=-2$. 

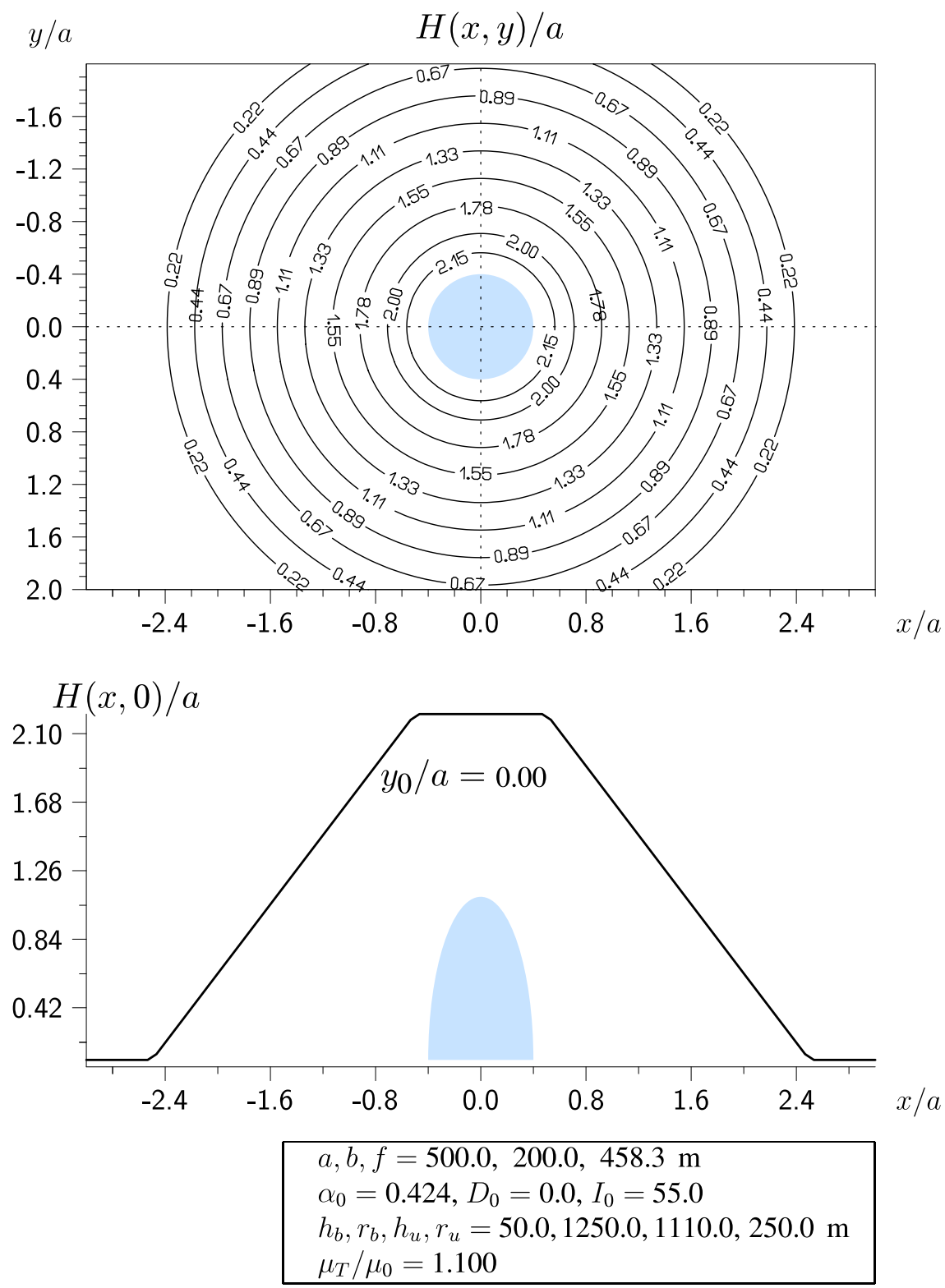

Fig. 3a. Isolines of the surface of the cut cone $H(x, y)=-Z(x, y)$ defined by equation (43) which represents hill above the prolate spheroidal magnetic body. The bottom curve shows hights profile at $y=0$. 

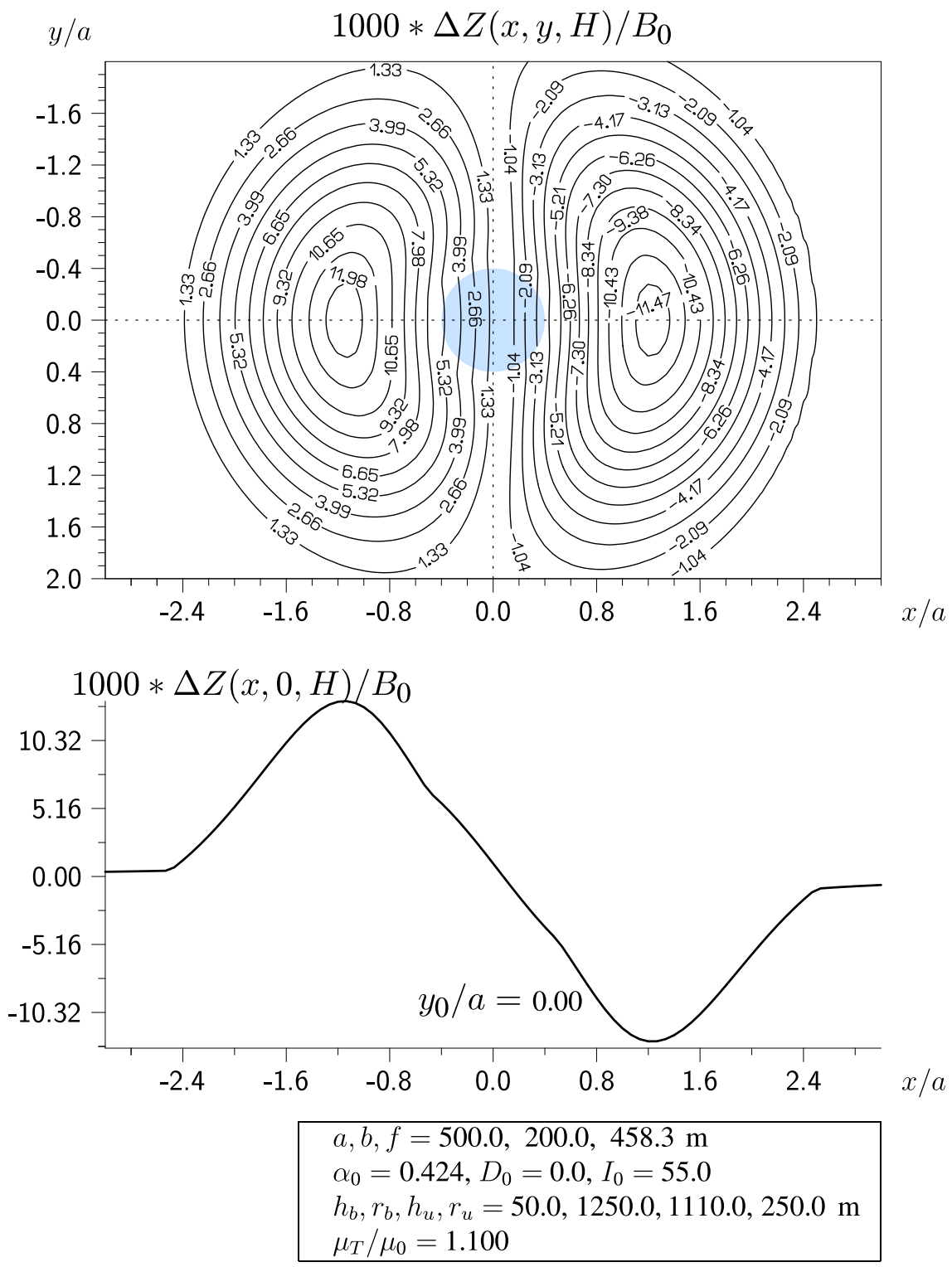

Fig. $3 \mathrm{~b}$. The isolines of the relative $\Delta Z$ anomaly above the prolate spheroidal body on the surface depicted in Fig. 3a. The bottom curve shows the profile values at $y=0$ along the hill. 

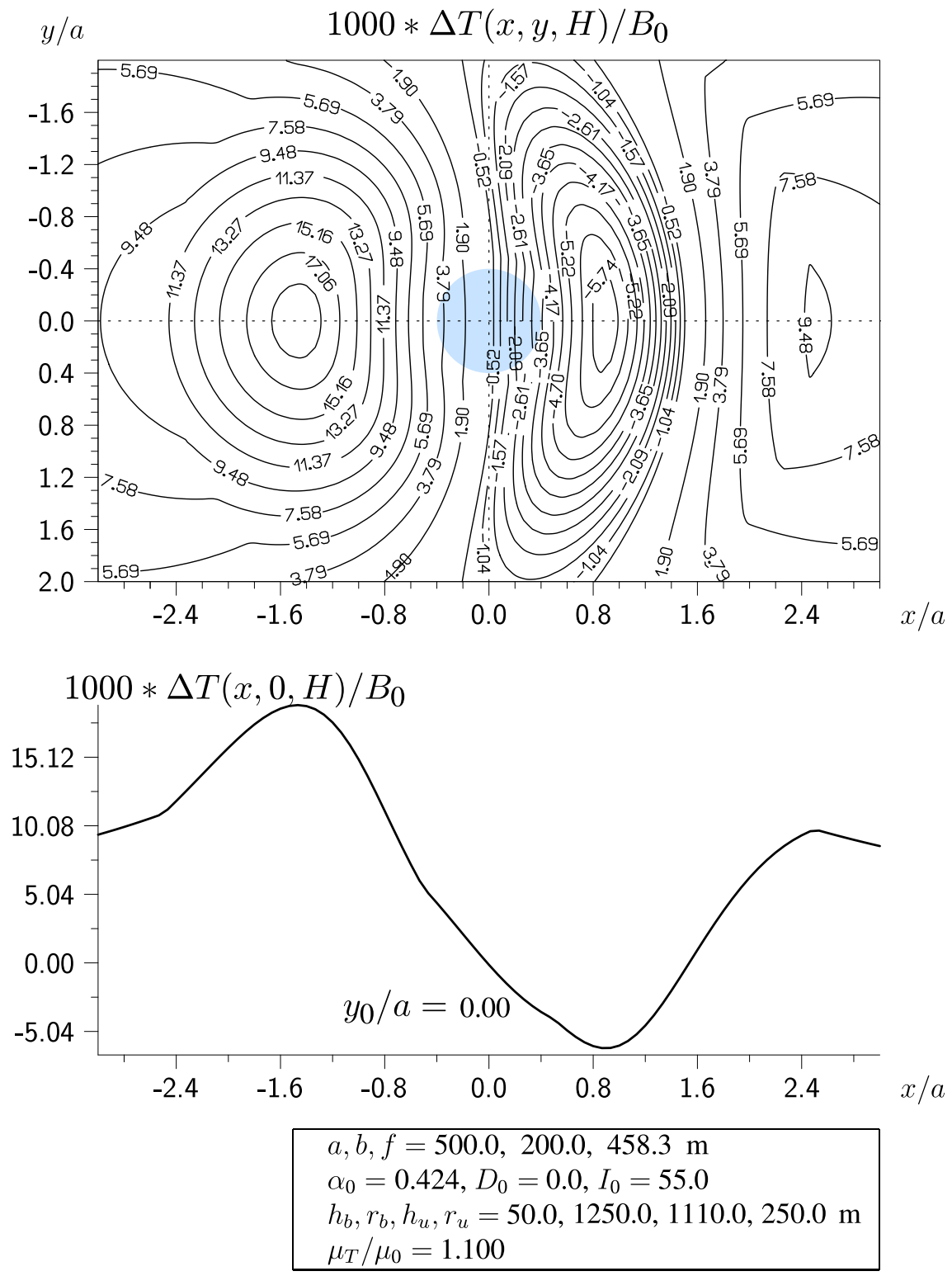

Fig. 3c. The isolines of the relative $\Delta T$ anomaly above the prolate spheroidal body on the surface depicted in Fig. 3a. The bottom curve shows the profile values at $y=0$ along the hill. 

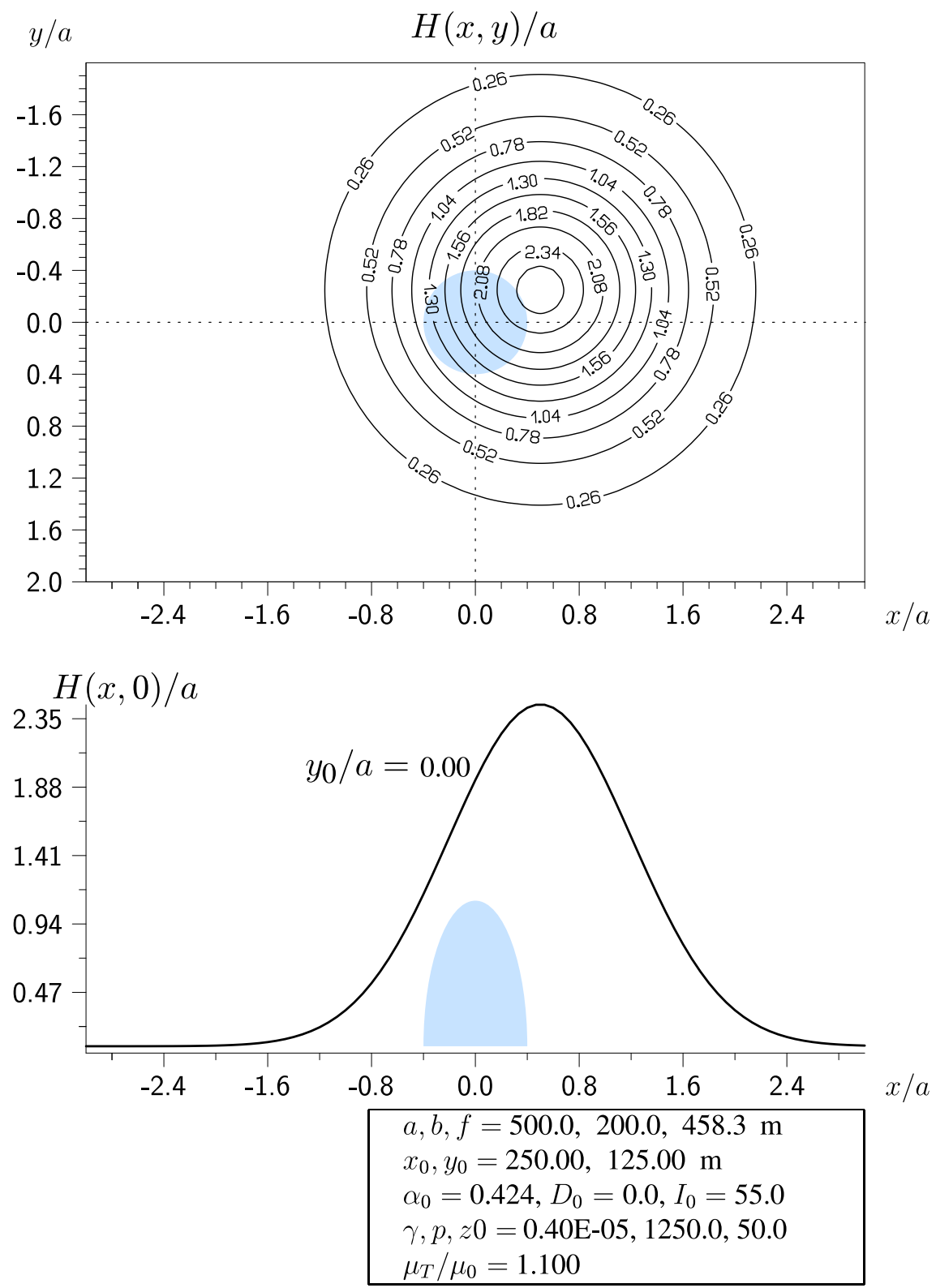

Fig. 4a. Isolines of the surface for the "Gaussian hill" $H(x, y)=-Z(x, y)$ defined by equation (44) which represents the eccentric hill above the prolate spheroidal body. The bottom curve shows hights profile at $y=0$. 

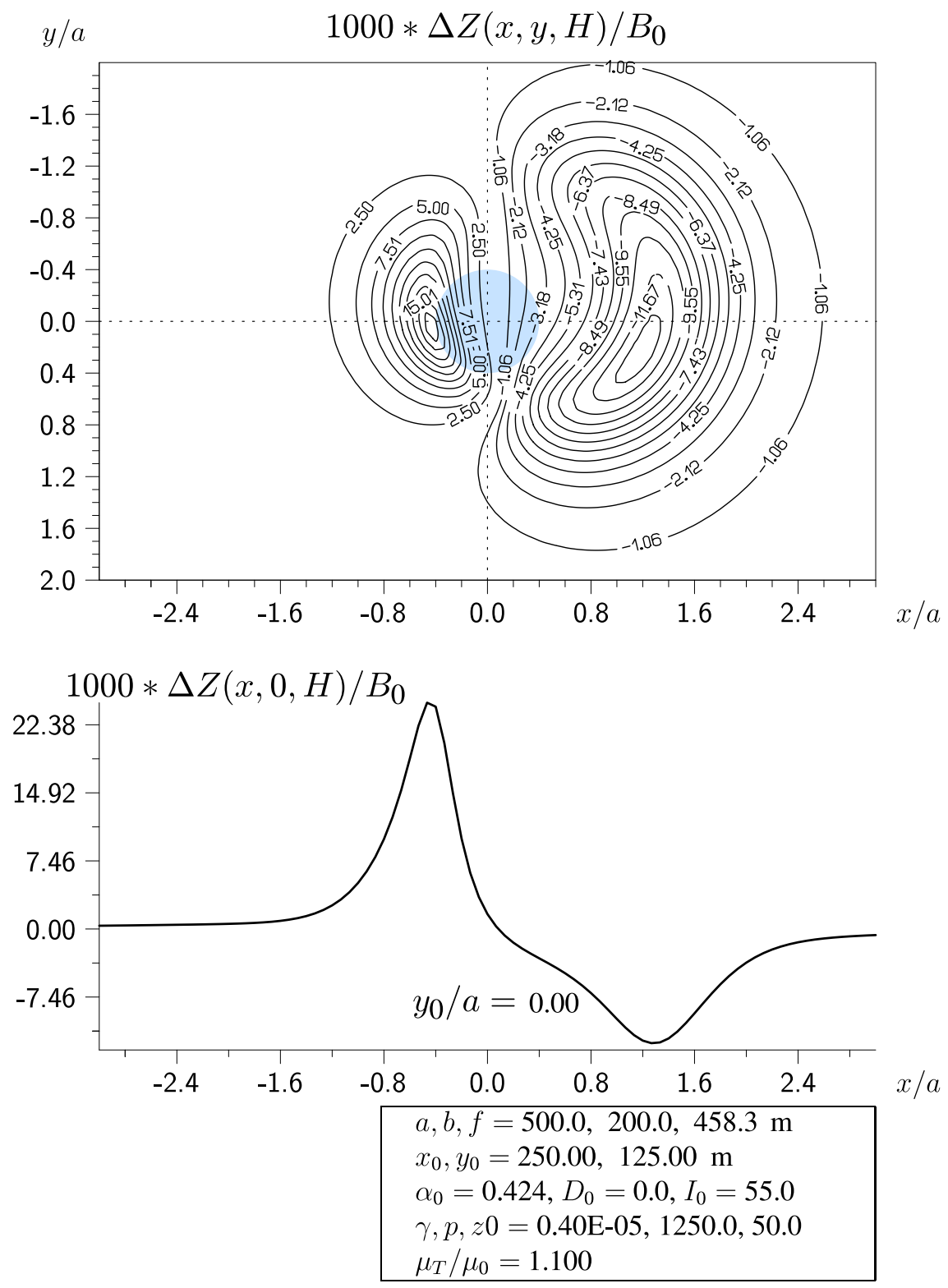

Fig. $4 \mathrm{~b}$. The isolines of the relative $\Delta Z$ anomaly above the prolate spheroidal body on the surface of the hill depicted in Fig. 4a. The bottom curve shows the profile values at $y=0$ along the hill. 

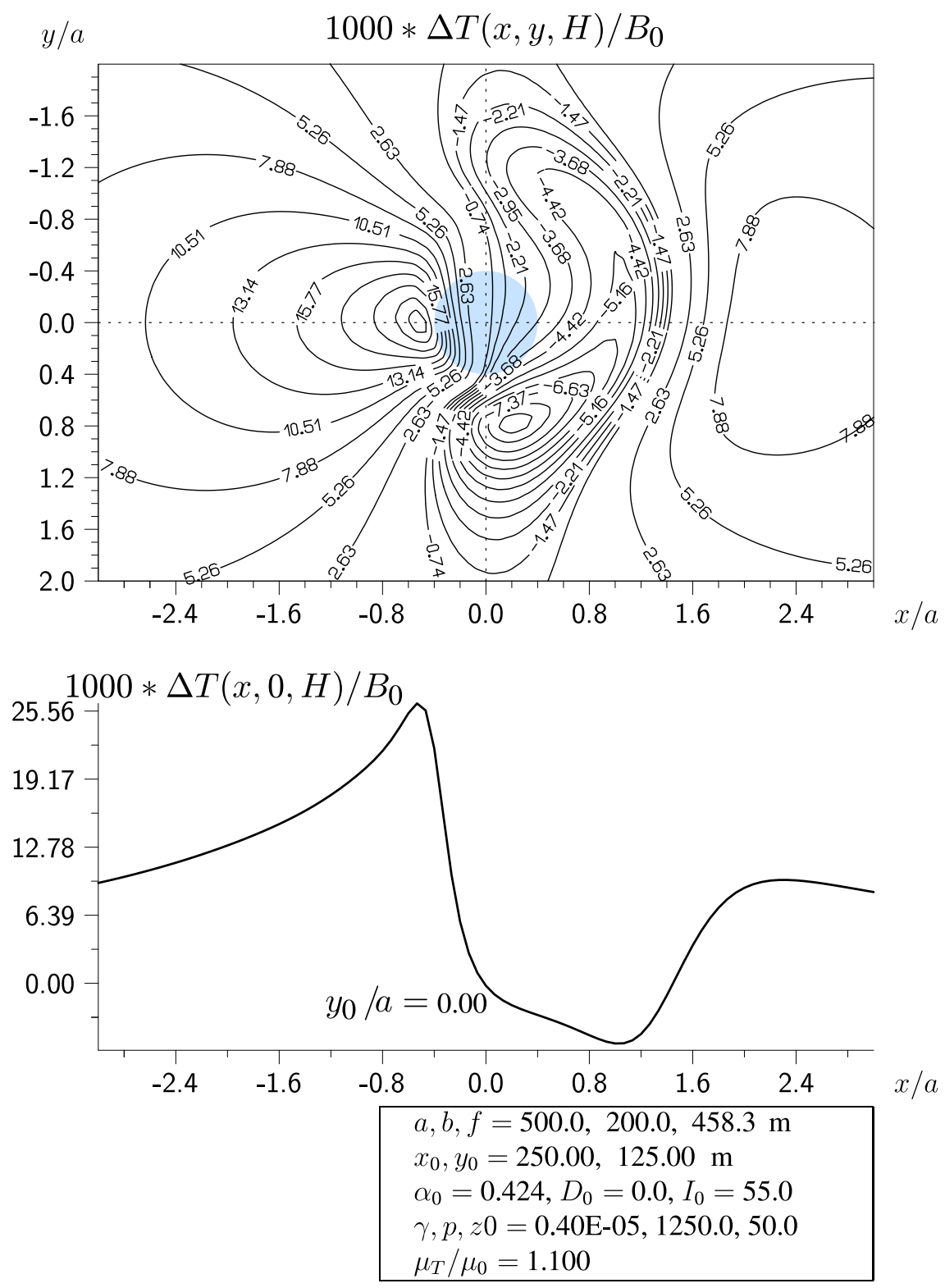

Fig. 4c. The isolines of the relative $\Delta T$ anomaly above the prolate spheroidal body on the surface of the hill depicted in Fig. 4a. The bottom curve shows the profile values at $y=0$ along the hill. 

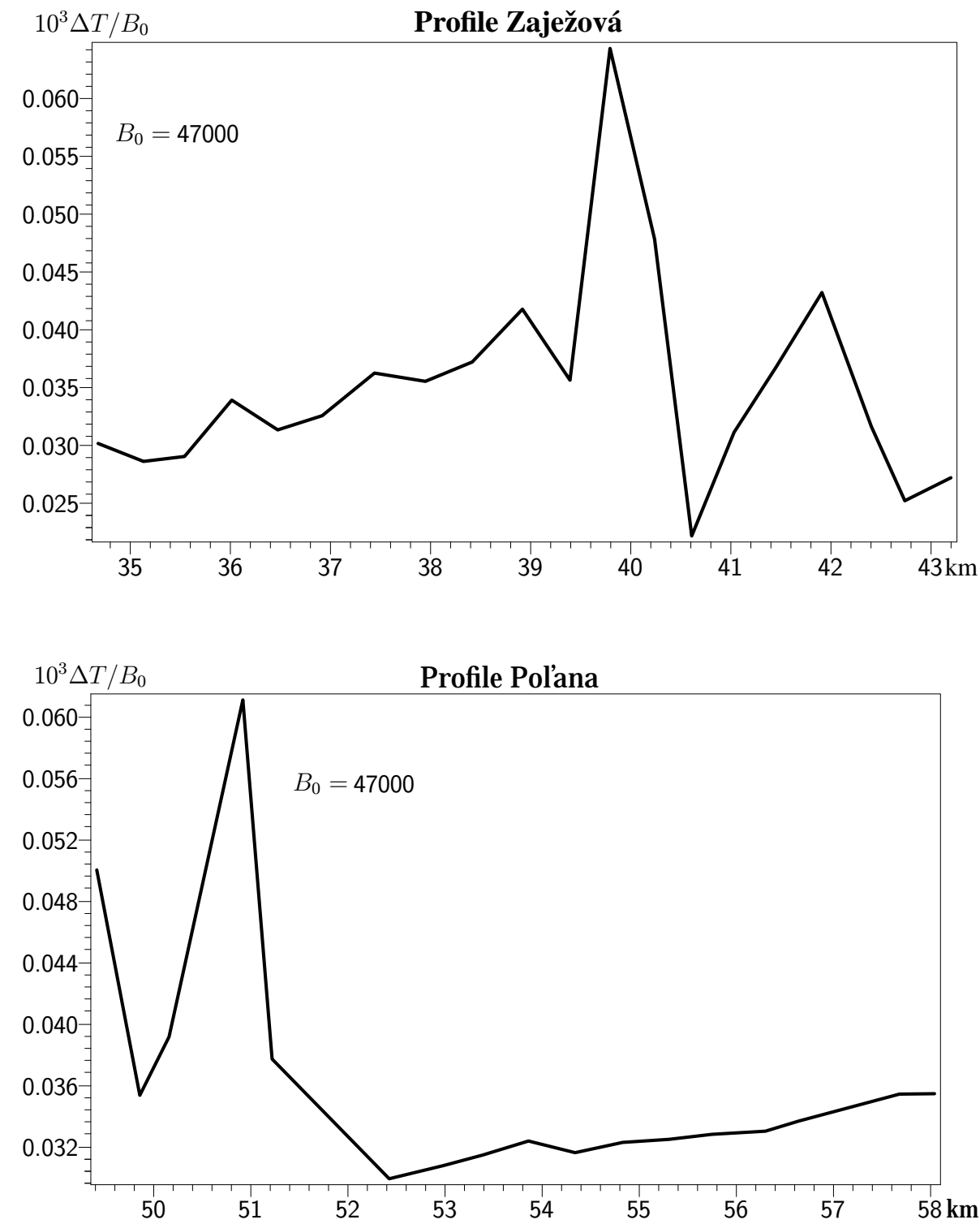

Fig. 5a,b. Values of $\Delta T / B_{0} \times 1000$ measured on two profiles (a - Zaježová, b - Polana) in the region of neovolcanites in the central Slovakia. 
occurs in the Mediterrean countries. For the ground magnetic survey on the volcanic hill we consider at first the broken line $Z$ profile ("hill profile"), which approximates the cut cone:

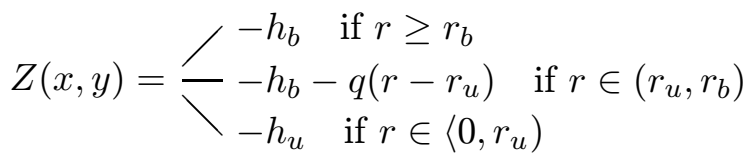

while $r=\sqrt{x^{2}+y^{2}}$ is the horizontal distance from the vertical axis of symmetry $z$. In Fig. 3a there are plotted hight isolines (isohypses) $H(x, y)=$ $-Z(x, y)$ defined by $(43)$ and at the bottom there is plotted a hight profile, as well as the position of the upper half of the magmatic prolate spheroid. Numerical values of parameters of the body and the "hill profile" are given in the Table in Fig. 3a. In Figs. 3b,c there are plotted isolines of $\Delta Z$ and $\Delta T$ values for this hill model. We can see that the changes of these fields are steeper in comparison to Figs. 2b,c. The extreme values are also higher. The next case of the "hill profile" is given by the Gaussian curve

$Z(x, y)=-z_{0}-p \exp \left\{-\gamma\left[\left(x-x_{0}\right)^{2}+\left(y-y_{0}\right)^{2}\right]\right\}$,

where we put: $z_{0}=a / 10, p=2.5 a, \gamma=1 / a^{2}$. The top of this hill is above the point $\left(x_{0}, y_{0}\right)$. The profile of this hill can be seen in Fig. 4a. When comparing with the previous hill case, we see that measuring points are closer to the anomalous body in flanks slopes. The top points are more distant. The isolines and profile graphs of the calculated values $\Delta Z$ and $\Delta T$ are presented in Figs. 4b,c. We can see that the extreme values are greater than in the previous hill model (Fig. 3a), clearly because the flank points of the "Gaussian hill" are closer to the anomalous body. In this manner we have obtained useful analytical model of sill-like magmatic bodies in volcanic hills.

We present also two practical profile curves of the $\Delta T / B_{0}$ obtained by the field measurements performed in the region of neovolcanites in the central Slovakia. Figure 5a concerns the profile at Zaježová in the Javorie Mts., while Fig. 5b shows the region beside the extinct stratovolcano Polana. We can see that the general source of these profile curves is in good agreement with theoretical profile curves $\Delta T / B_{0}$, although the relative anomaly values are about 1000 times less. This can be caused by the two main reasons: 
i) the susceptibility $\kappa$ in our theoretical calculations was put very high $\kappa=0.1$ SI units, while in real conditions we can have $\kappa \approx 10^{-4} \mathrm{SI}$,

ii) the measurement profiles for Figs. 5a,b do not run across the top of the volcanic hill, but about $1-3 \mathrm{~km}$ aside.

Acknowledgments. The author is grateful to the Slovak Grant Agency VEGA for partial support of the work by means of project No. 2/0169/09 and to the APVV Grant Agency, project No. 51-008505.

\section{References}

Arfken G., 1966: Mathematical methods for physicists, Academic Press London, 1966.

Cook K. L., Van Nostrand R. C., 1954: Interpretation of resistivity data over filled sinks. Geophysics, 19, 761-770.

Hvoždara M., 2008: Groundwater and geothermal anomalies due to a prolate spheroid. Contrib. Geophys. Geod., 39, 2, 95-119.

Lebedev N. N., 1963: Special functions and their applications (in Russian). Fizmatgiz, Moscow.

Madelung E., 1957: Die Mathematischen Hilfsmittel des Physikers. Spriger-Verlag, Berlin. Morse P. M., Feshbach H., 1973: Methods of theoretical physics. McGraw-Hill Comp., New York.

Smythe W. R., 1968: Static and dynamic electricity. McGraw-Hill, New York.

Wait J. R., 1982: Geo-electromagnetism. Academic Press, New York. 